This item was submitted to Loughborough's Research Repository by the author.

Items in Figshare are protected by copyright, with all rights reserved, unless otherwise indicated.

\title{
Ultra thin dipole antenna backed by new planar artificial magnetic conductor
}

PLEASE CITE THE PUBLISHED VERSION

http://dx.doi.org/10.1109/LAPC.2009.5352575

PUBLISHER

Loughborough University (@ IEEE)

VERSION

AM (Accepted Manuscript)

LICENCE

CC BY-NC-ND 4.0

REPOSITORY RECORD

Al-Nuaimi, M.K.T., and W.G. Whittow. 2019. "Ultra Thin Dipole Antenna Backed by New Planar Artificial Magnetic Conductor". figshare. https://hdl.handle.net/2134/10000. 
This item was submitted to Loughborough's Institutional Repository (https://dspace.lboro.ac.uk/) by the author and is made available under the following Creative Commons Licence conditions.

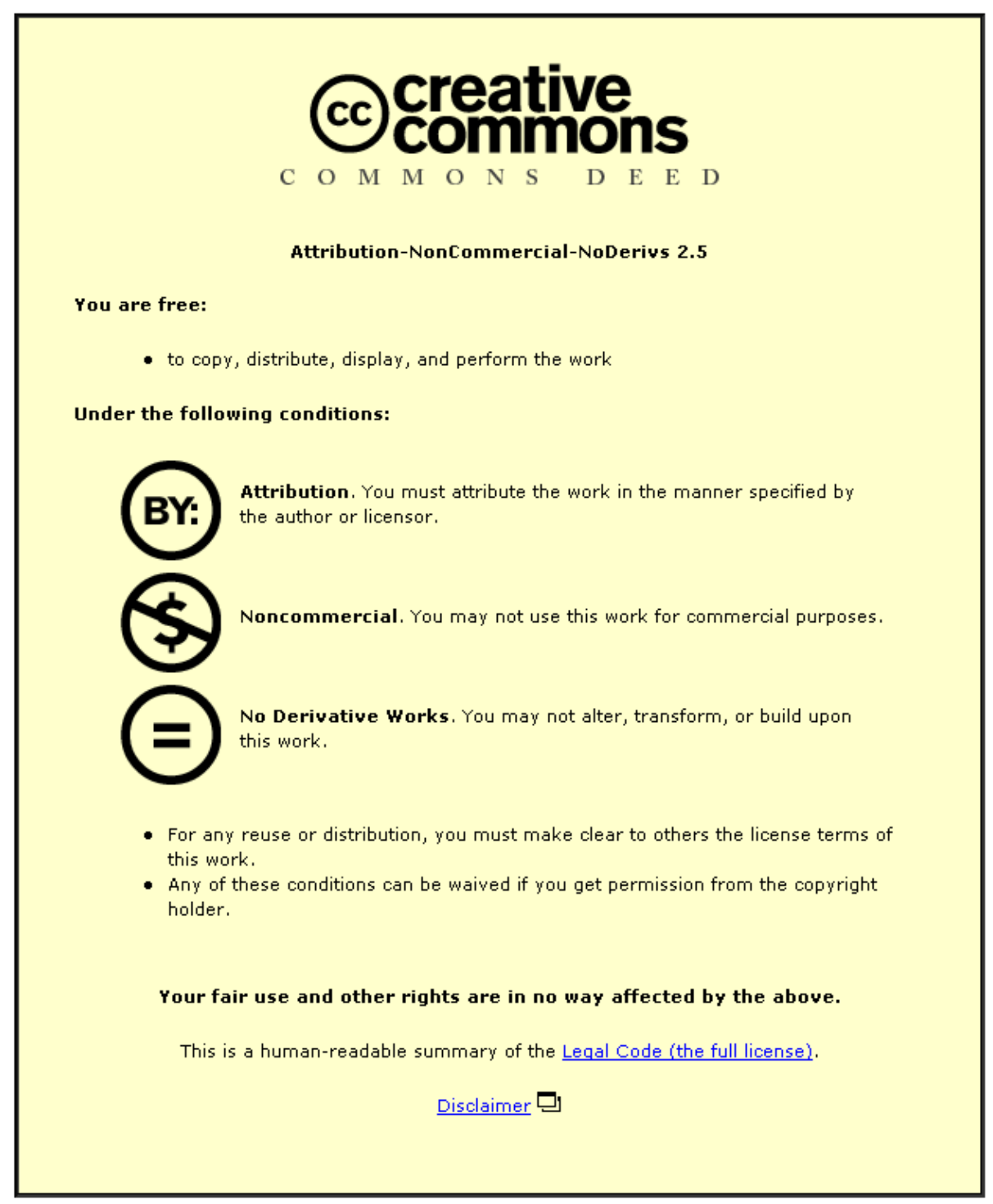

For the full text of this licence, please go to: http://creativecommons.org/licenses/by-nc-nd/2.5/ 


\title{
Ultra Thin Dipole Antenna Backed by New Planar Artificial Magnetic Conductor
}

\author{
Mustafa K. Taher Al-Nuaimi ${ }^{\# 1}$ and William G. Whittow ${ }^{* 2}$ \\ ${ }^{\#}$ Department of Electrical and Electronic Engineering, University of Technology, Baghdad, IRAQ \\ mustafa.engineer@yahoo.com \\ ${ }^{*}$ Department of Electronic and Electrical Engineering, Loughborough University, Loughborough, UK \\ w.g.whittow@lboro.ac.uk
}

\begin{abstract}
In some wireless communications it is desirable for antennas to have a low profile configuration. In such a design, the overall height of the antenna structure is usually less than one tenth of the operating wavelength. A fundamental challenge in low profile wire antenna design is the coupling effect of a nearby ground plane. A compact highly directional dipole antenna backed by our novel proposed planar artificial magnetic conductor (AMC) structure with an overall height of $0.034 \lambda$ is investigated. Two different dipoles are used in this study, wire and printed dipole antenna over the proposed AMC. A comparative study is conducted to study the return loss of the dipole antenna parallel above the proposed $A M C$ and the ideal perfect electric conductor (PEC). A numerical simulation model using Ansoft high frequency structure simulator (AnsoftHFSS $^{\mathrm{TM}}$ ) is developed to predict the reflection phase stop band of the proposed structure. The peak gain of the antenna over the proposed $\mathrm{AMC}$ is close to $8 \mathrm{dBi}$.
\end{abstract}

\section{INTRODUCTION}

Electromagnetic band-gap (EBG) structures have recently attracted a great deal of interest among researchers to design and develop low profile antennas. Special importance has been placed on thin lightweight directive dipole antennas that are greatly desired in commercial and defence wireless applications. Two interesting features associated with EBG structures are suppression of surface waves and in-phase reflection coefficient for plane waves [1-3]. When the incident wave is a surface wave, the EBG structure shows a frequency band gap through which the surface waves cannot propagate regardless of incident angles and polarization states, resulting in an ideal isolator for electromagnetic waves [4]. Suppression of surface waves results in higher efficiency, smoother radiation pattern, and less back lobe and side lobe levels in antenna applications particularly for microstrip antennas [5-7]. In addition, the in-phase reflection property of these structures can be used in the design of low profile antennas because the radiating element can lie directly adjacent to the ground plane without being shorted. Electromagnetic Band gap (EBG) surfaces have been used in recent years to mimic a Perfect Magnetic Conductor (PMC) over a finite frequency range. This physical realization of a PMC, known as an Artificial
Magnetic Conductor (AMC). Dipole antennas do not function effectively when positioned very closely and parallel above a PEC (perfect electric conductor) ground plane due to the reverse image currents which reduce the antenna radiation efficiency. To overcome this difficulty dipoles are operated at a height of $0.25 \lambda$ or higher from the ground plane which is not practical for the wireless communication systems. Ideal PMC ground plane will create the positive image currents of the parallel dipole antenna above it. However, no natural material has ever been found to realize the magnetic conducting surface.

Due to the design complexity and cost factor associated with 3D mushroom type AMCs, researchers are focusing on planar AMC structures, such as the UC-PBG [8, 9]. Nevertheless, there is a clear need for smaller unit cell planar AMC structures to support various wireless applications. In this paper, we propose a smaller unit cell planar AMC geometry with a significantly smaller unit cell size without via connections as in the mushroom like EBG and smaller in size compared to the UC-PBG case. Here we focus on designing ultra thin directional wire and printed dipole antennas $(0.034 \lambda)$ based on the principles AMC.

\section{EBG REFLECTION PHASE CHARACTERISTICS}

In order to achieve good return loss and high efficiency, low profile antennas are generally placed horizontally close to an AMC surface which is designed in such a manner that the zero degree AMC reflection phase frequency coincides with the operating frequency of the antenna. Therefore, determining the reflection phase characteristics of the proposed AMC structure is important. Figure 1 (a) shows a unit cell of the proposed AMC. The HFSS model to compute the reflection phase characteristics of the proposed AMC structure is shown in Fig. 1 (b). This model is based on simulating scattering parameters of a single port air filled waveguide with two perfect electric conductor (PEC) and two perfect magnetic conductor (PMC) walls. The propagating plane wave is polarized parallel to the PMC walls and normal to the PEC walls. The waveguide is then terminated to a single unit cell of the proposed AMC structure and the reflection 
Final author version. Loughborough Antennas and Propagation Conference, LAPC 2009, Loughborough, UK Conference Proceedings. PP 97-100. 2009

phase is obtained by calculating the scattering parameter at the input of this single port waveguide. There are several methods for computing the AMC reflection phase [10, 11]. Comparing to conventional methods, the main advantage of this model is its simplicity. However, the model also shown to be fast and accurate.

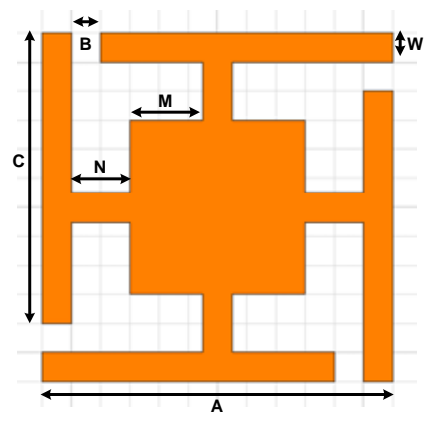

(a)

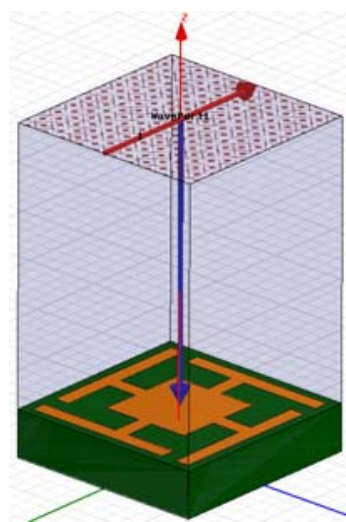

(b)
Fig. 1 (a) Unit cell of the proposed AMC structure with dimensions $A=6.6 \mathrm{~mm}$, $\mathrm{C}=5 \mathrm{~mm}, \mathrm{M}=1.25 \mathrm{~mm}, \mathrm{~N}=1 \mathrm{~mm}, \mathrm{w}=0.5 \mathrm{~mm}, \mathrm{~B}=0.5 \mathrm{~mm}$, substrate thickness $=$ $1.52 \mathrm{~mm}$ with $\varepsilon_{\mathrm{r}}=6.15$ (b) HFSS model for AMC reflection phase computation.

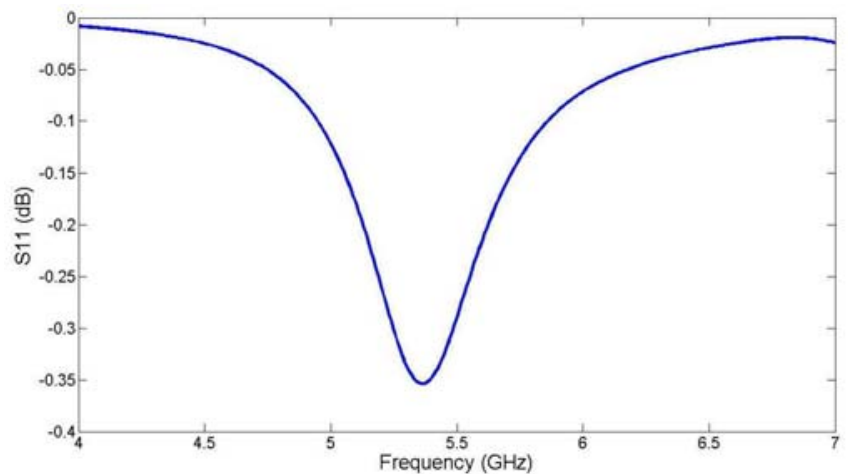

(a)

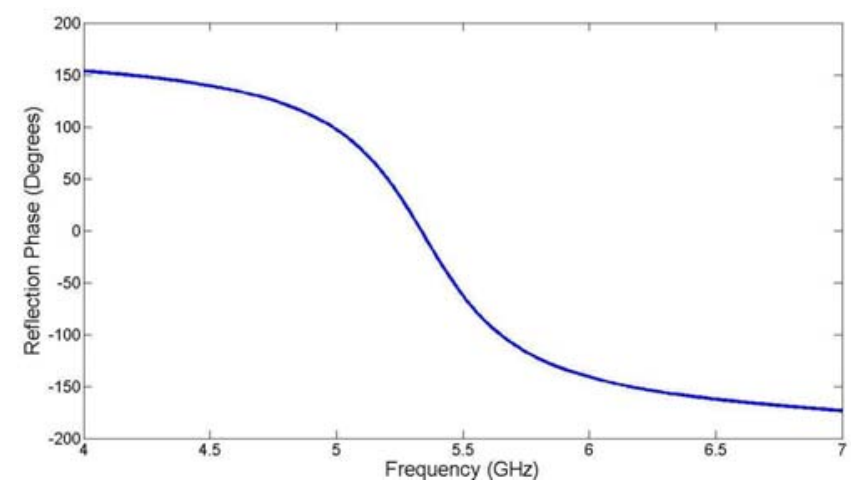

(b)

Fig. 2 HFSS simulation results (a) $S_{11}$ and (b) reflection phase of the proposed AMC.
Figure 2 (b) shows the computed reflection phase profile of the proposed planar AMC structure for a normally incident plane wave. It is known that a $\mathrm{PEC}$ has a $180^{\circ}$ reflection phase and a $\mathrm{PMC}$ has a $0^{\circ}$ reflection phase [12]. In contrast, the reflection phase of the AMC surface decreases continuously from $180^{\circ}$ to $-180^{\circ}$ as frequency increases. The proposed AMC surface exhibits a $90^{\circ}$ reflection phase around $5.1 \mathrm{GHz}$ and a $0^{\circ}$ reflection phase around $5.33 \mathrm{GHz}$. It is important to note that the reflection phase varies with incident angles and polarization states.

\section{DiPOLE ANTENNA OVER AMC STRUCTURE}

The complete antenna system including the dipole antenna and AMC ground plane was simulated using ANSOFT$\mathrm{HFSS}^{\mathrm{TM}}$. To illustrate the in-phase reflection feature of the proposed planar AMC a cylindrical dipole of $0.5 \mathrm{~mm}$ radius was simulated above the PEC and AMC ground planes. The overall height of the dipole antenna from the bottom conductor of the proposed AMC structure is $0.034 \lambda_{5.1 \mathrm{GHz}}$. The height of dipole from the top surface of the proposed AMC structure is $0.0085 \lambda_{5.1 \mathrm{GHz}}$ as shown in Fig. 3 and Fig. 4.

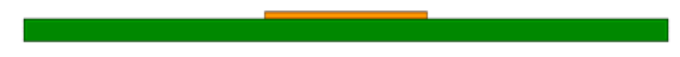

(a)

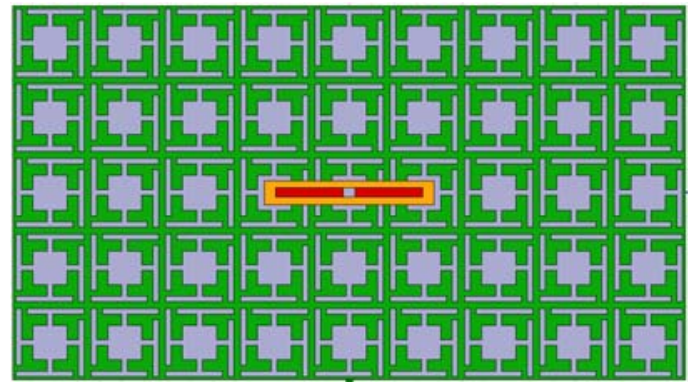

(b)

Fig. 3 Printed dipole antenna over AMC ground plane (a) side view (b) top view.

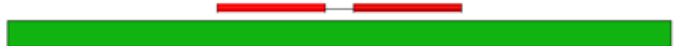

(a) 
Final author version. Loughborough Antennas and Propagation Conference, LAPC 2009, Loughborough, UK Conference Proceedings. PP 97-100. 2009

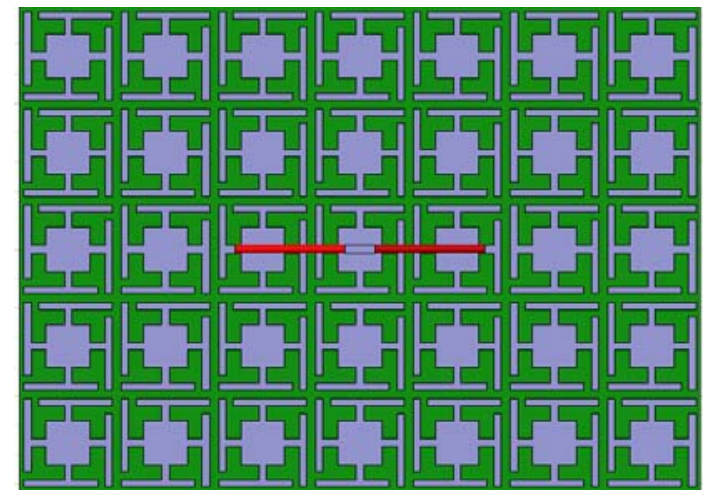

(b)

Fig. 4 Wire dipole antenna over AMC ground plane (a) side view (b) top view.

In the printed dipole case, the dipole was printed on a $0.5 \mathrm{~mm}$ thin RT/Duroid 5880 substrate with $\varepsilon_{\mathrm{r}}=2.2$. The computed return loss data for the wire and printed dipoles are shown in Fig. 5 and Fig. 6 respectively for different antenna lengths.

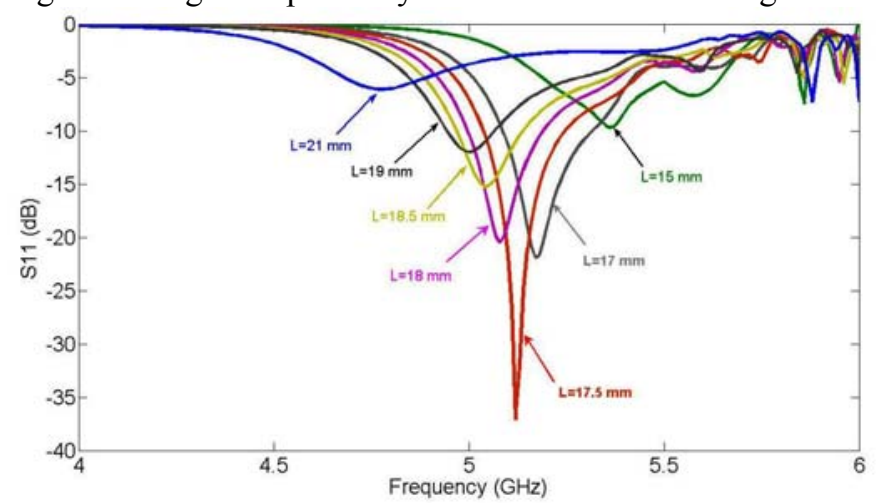

(a)

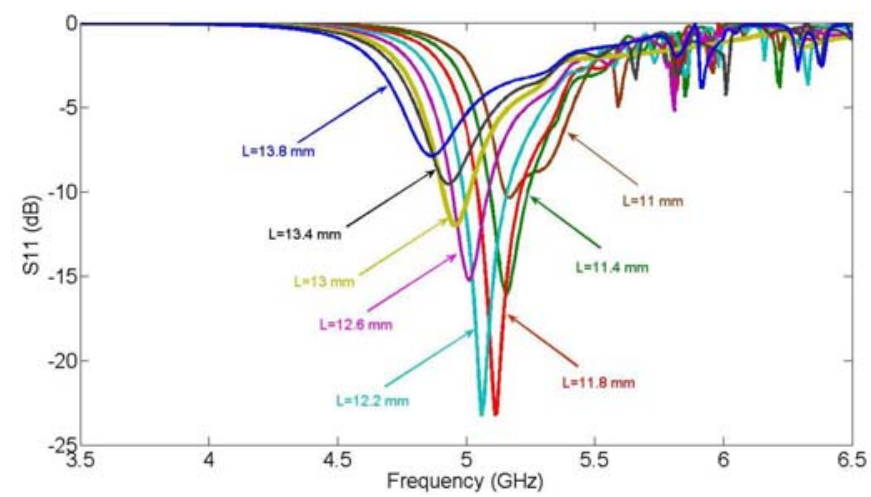

Fig. 6 (a)Return loss values of the printed dipole antenna over AMC for different antenna lengths (b) Return loss values of the wire dipole antenna over AMC for different antenna lengths

Figure 7 compares the return loss of the wire dipole and printed dipole antennas over the proposed AMC and PEC respectively. The dipole on top of a PEC placed at the same height demonstrates a poor return loss performance. In contrast, when the dipole is placed on top of the planar AMC structure, the S-parameters clearly shows the presence of the resonance due to the AMC structure. When the PEC surface is used as the ground plane, the return loss $\left(\mathrm{S}_{11}\right)$ of the dipole is about $-0.1 \mathrm{~dB}$. The reason is that the PEC surface has a $180^{\circ}$ reflection phase and the image current has an opposite direction to that of the original dipole. The reverse image current cancels the radiation of the dipole, resulting in a very poor return loss. The best return loss of $-38 \mathrm{~dB}$ and $-24 \mathrm{~dB}$ is achieved by the dipole antenna over the proposed $\mathrm{AMC}$ ground plane. The reflection phase of the AMC surface varies from $180^{\circ}$ to $-180^{\circ}$ with frequency. In a certain frequency band, the AMC surface successfully serves as the ground plane of the low profile dipole so that the dipole antenna can radiate efficiently. From this comparison it can be seen that the AMC surface is a good ground plane candidate for low profile wire antenna designs. The steepness of the planar AMC reflection phase curve [see Fig. 2] contributes to relatively narrow antenna bandwidth. The peak gain of the dipole antenna at $5.1 \mathrm{GHz}$ is close to $8 \mathrm{dBi}$.

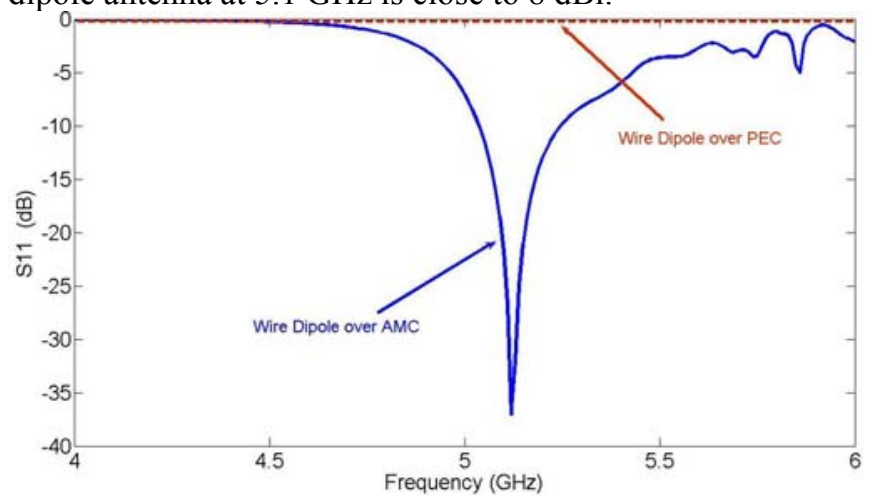

(a)

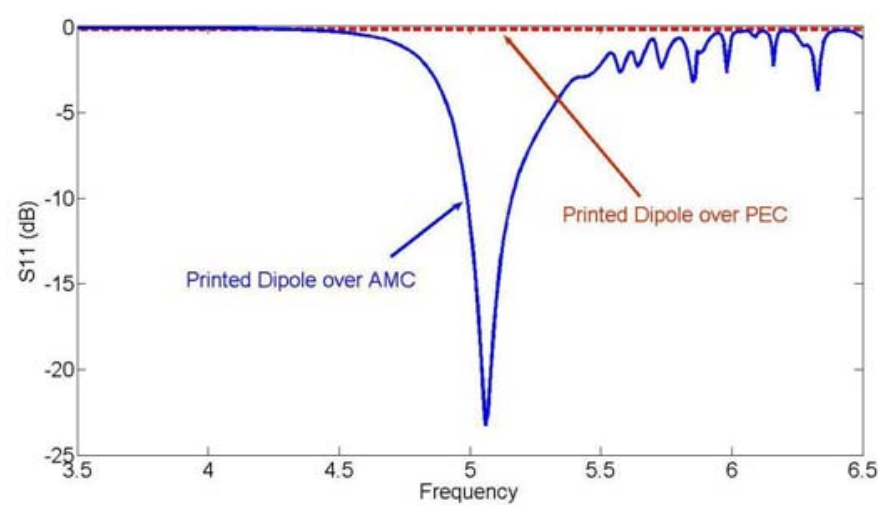

(b)

Fig. 7 Return loss of the (a) wire dipole and (b) printed dipole over both AMC and PEC respectively.

\section{CONCLUSIONS}

An ultra thin directive wire and printed dipole antenna backed by a new AMC structure is investigated. The in-phase reflection feature of our proposed planar AMC is demonstrated by developing an ultra-thin $(0.034 \lambda)$ cylindrical dipole antenna. The proposed AMC structure needs less 
Final author version. Loughborough Antennas and Propagation Conference, LAPC 2009, Loughborough, UK Conference Proceedings. PP 97-100. 2009

complexity in the fabrication since it's via less structure compared to the mushroom like structure and smaller in size compared to the UC-PBG.

\section{REFERENCES}

[1] N. Engheta, R. W. Ziolkowski,'Electromagnetic Metamaterials: Physics and Engineering Explorations", Wiley interscience, 2006.

[2] F. Yang, Y. R. Samii,"Electromagnetic Band Gap Structures in Antenna Engineering", Cambridge University Press, 2009.

[3] J. Volakis," Antenna Engineering Handbook", McGraw-Hill Professional, Fourth Edition, 2007.

[4] D. Sievenpiper, L. Zhang, R. F. Broas, N. G. Alex'opolous, and E. Yablonovitch, "High-Impedance Electromagnetic Surfaces with a Forbidden Frequency Band", IEEE Transactions On Microwave Theory And Techniques, Vol. 47, No. 11, November 1999.

[5] F. Yang and Y. R. Samii, "Microstrip Antennas Integrated With Electromagnetic Band-Gap (EBG) Structures: A Low Mutual Coupling Design for Array Applications", IEEE Transactions on Antennas and Propagation, Vol. 51, No. 10, October 2003.

[6] F. Yang and Y. R. Samii, "Wire Antenna On An EBG Ground Plane Vs. Patch Antenna: A Comparative Study On Low Profile Antennas",
Electromagnetic Theory Symposium, July 26-28, Ottawa, Canada 2007

[7] QU D., Shafai L. and A. Foroozesh, "Improving Microstrip Patch Antenna Performance Using EBG Substrates", IEE Proc.-Microw. Antennas Propag., Vol. 153, No. 6, December 2006

[8] C. C. Chang, Y. Qian, and T. Itoh ,"Analysis And Applications Of Uniplanar Compact Photonic Bandgap Structures", Progress In Electromagnetics Research, PIER 41, 211-235, 2003.

[9] F. Yang, K. Ma, Y. Qian, and T. Itoh,"A Uniplanar Compact Photonic-Bandgap (UC-PBG) Structure and Its Applications for Microwave Circuits", IEEE Transactions on Microwave Theory and Techniques, Vol. 47, No. 8, August 1999.

[10] Fan Yang and Yahya Rahmat-Samii,"Reflection Phase Characterization of an Electromagnetic Band-Gap (EBG) Surface", Antennas and Propagation Society International Symposium, 2002.

[11] F. Yang and Y. Rahmat-Samii, "Reflection phase characterizations of the EBG ground plane for low profile wire antenna applications", IEEE Trans. Antennas Propagation., vol. 51, no. 10, pp. 2691-2703, Oct. 2003.

[12] Balanis, C.A.,"Antenna Theory: Analysis and Design", John Wiley \& Sons, Inc, 2005. 